\title{
Elemental, configural, and sequential memory processes in the rat can be tested in a single situation in one day
}

\author{
YOSHIO SAKURAI \\ Kyoto University, National Institute of Physiological Sciences, and PRESTO, Kyoto, Japan
}

\begin{abstract}
The present study shows that rats can perform behavioral tasks that test their elemental auditory, elemental visual, configural auditory-visual, and sequential auditory-visual memory processes. All the tasks employed an identical apparatus and identical stimuli and differed only in which feature of the stimuli was to be processed for correct performance. The rat made go or no-go responses to indicate whether the presented stimulus included specific tones (elemental auditory), lights (elemental visual), overlapped tones and lights (configural auditory-visual), or sequential tones and lights (sequential auditory-visual). The rats came to perform well in all the tasks in 1 day. Probe sessions proved that the rats did indeed discriminate elemental stimuli in the elemental tasks. This method makes it possible to compare activities from the same individual neurons among different memory processes.
\end{abstract}

The most extreme views concerning the neuronal code of information processing are the single-neuron coding and the population ensemble coding hypotheses (Sakurai, 1996b). The former is based on the single-neuron doctrine (Barlow, 1972), which holds that behavioral functions are uniquely encoded in the activity of individual neurons. The latter view is based on the theory of the cell assembly (Hebb, 1949), which is a set of coactive neurons that are involved in several information processes.

The coding by cell assemblies has two major properties (Palm, 1990; Sakurai, 1998a, 1998b). The first one is the neuron-overlapping property-that is, the same neuron is a part of different assemblies underlying different information processes. The second one is the connectiondynamics property - that is, the functional synaptic connections needed to form different cell assemblies change dynamically among different information processes. An adequate experimental strategy to test these properties is "a multiple-task comparison of individual neuronal activities and multiple neuronal correlations" (Sakurai, 1999, p. 787). The key point here is that the same neurons are recorded during different behavioral tasks. There have already been several papers in which the dynamical aspects of neuronal ensemble coding during different tasks or different components of a complex task have been examined (e.g., Gothard, Skaggs, Moore, \& McNaughton, 1996; Tanila, Shapiro, Gallagher, \& Eichenbaum, 1997).

This work was supported by Grants-in-Aid for Scientific Research (10164228 and 09610076) from the Japanese Ministry of Education, Science and Culture and by the "Research for the Future" Program (96L00206) from the Japan Society for the Promotion of Science. Correspondence concerning this article should be addressed to Y. Sakurai, Department of Psychology, Graduate School of Letters, Kyoto University, Yoshida-honmachi, Sakyo-ku, Kyoto 606-8501, Japan (e-mail: sakurai@psy.bun.kyoto-u.ac.jp).
The present study introduces adequate behavioral tasks with which to examine different information processings of memory in the rat in a short period (1 day), during which time the neuronal activity of identical neurons can be recorded in different tasks more easily. All the tasks employed identical stimuli, apparatus, and response modes and differed only in which features-elemental, compound, or sequential - of the stimuli were to be processed on the basis of memory. This type of battery of tasks could give a more adequate multiple-task comparison of individual neuronal activities and multiple neuronal correlations, in order to reveal the modes of neuronal coding underlying the information processing, not of physical, but of psychological features of stimuli in the working brain (Sakurai, 1993, 1994). The present study also conducted probe sessions of the tasks, which tested whether the rats did, in fact, process the different features of the stimuli among the different behavioral tasks.

\section{METHOD}

\section{Subjects}

A total of ten 5-month-old male albino rats (Sankyo Labs, Toyama) was used. The rats were individually housed, with a 12:12$\mathrm{h}$ light:dark cycle. The animal room was maintained at $24^{\circ} \pm 2^{\circ} \mathrm{C}$. Cage dimensions were $25 \times 15 \times 20 \mathrm{~cm}$. Lights were on from 9:00 a.m. to 9:00 p.m. All behavioral training and recording were conducted during the light phase of the cycle. All the rats were fed enough lab chow $1-3 \mathrm{~h}$ after their daily training or recording sessions to maintain them at $90 \%$ of their ad-lib weight. Water was available continuously.

\footnotetext{
Apparatus

Behavioral training took place in an operant chamber, $23 \times 11 \times$ $35 \mathrm{~cm}$ (Sakurai, 1990). One wall of the chamber had a translucent response panel, $4 \times 7 \mathrm{~cm}, 3 \mathrm{~cm}$ above the floor. The panel was illuminated by an $8-\mathrm{V}$, direct current bulb, which was covered by a guillotine door. A buzzer was located behind the panel. A loudspeaker
} 


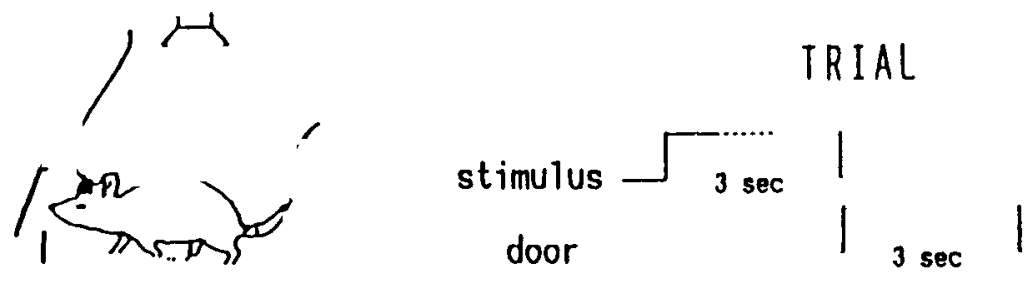

\section{Elemental Auditory Discrimination}

\section{Elemental Visual Discrimination}

$A+X \rightarrow G O$
$A+Y$
$B+X \rightarrow G O$
$B+Y$

\section{Configural Auditory-Visual Discrimination}

\section{Sequential Auditory-Visual Discrimination}

$$
\begin{aligned}
& A+X \rightarrow G O \\
& A+Y \rightarrow G O \\
& B+X \\
& B+Y
\end{aligned}
$$

$A+X \rightarrow G$ G
$A+Y$
$B+X$
$B+Y$

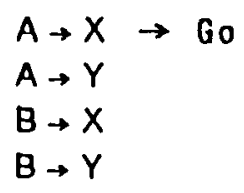

Figure 1. Top left panel: the apparatus for the tasks. Top right panel: sequence of events within a trial. Bottom panel: the discriminative stimuli and correct responses in each of the tasks.

( $15 \mathrm{~cm}$ in diameter) was set $30 \mathrm{~cm}$ above the top of the chamber for presentation of auditory stimuli, and two $8-\mathrm{V}$, direct current bulbs were placed $2 \mathrm{~cm}$ to the left and right of the chamber for presentation of the visual stimuli. A food dispenser delivered a $45-\mathrm{mg}$ food pellet to a magazine that was located $1.5 \mathrm{~cm}$ above the floor and $1 \mathrm{~cm}$ from the corner of the right wall. The chamber was enclosed in a soundproof box (Japan Shield Enclosure, Osaka)

\section{Behavioral Tasks}

Common procedure of all the tasks. The auditory stimuli were high tones $(10 \mathrm{kHz}, 85 \mathrm{~dB}$; tone $\mathrm{A})$ and low tones $(2 \mathrm{kHz}, 85 \mathrm{~dB}$; tone $B$ ) from the loudspeaker. The visual stimuli were illuminations of the right-side bulb (light $X$ ) and the left-side bulb (light $Y$ ). The discriminative stimuli in the tasks were $\mathrm{AX}, \mathrm{AY}, \mathrm{BX}$, and $\mathrm{BY}$ - that is, four stimuli, each of which was composed of one auditory and one visual stimulus. At each trial, one of the discriminative stimuli was presented and continued for $3 \mathrm{sec}$. Following stimulus offset, the guillotine door opened to make the illuminated response panel available for $3 \mathrm{sec}$. Pressing the panel during the 3-sec period was a go response, and not pressing it for the 3-sec period was a no-go response. A correct go response turned the panel light off and delivered a food pellet $(45 \mathrm{mg})$. An incorrect go response turned the panel light off, produced a 0.5 -sec buzzer noise, and was followed by 2 further trials with the same discriminative stimulus (correction trials), on which no-go responses were required. No data were obtained from the correction trials. Only correct go responses delivered the food reward (asymmetrical reward). The intertrial interval was $12 \mathrm{sec}$. A session consisted of 100 trials for each task, not including correction trials. The numbers of trials with each discriminative stimulus in a session were counterbalanced to make the ratio of reward and nonreward trials 2:3 in each session. Such unbalanced ratios of reward and nonreward trials have been shown to be effective for the training of memory tasks (Pontecorvo, 1983; Sakurai, 1987, 1992).

Elemental auditory discrimination task. The discriminative stimuli were compound ones-that is, simultaneous overlapped presentations of auditory and visual stimuli $(\mathrm{A}+\mathrm{X}, \mathrm{A}+\mathrm{Y}, \mathrm{B}+\mathrm{X}$, $B+Y)$. Go responses to the discriminative stimuli that included tone
$\mathrm{A}(\mathrm{A}+\mathrm{X}, \mathrm{A}+\mathrm{Y})$ and no-go responses to the discriminative stimuli that included tone $\mathrm{B}(\mathrm{B}+\mathrm{X}, \mathrm{B}+\mathrm{Y})$ were correct. The rat was required to discriminate the elemental auditory stimuli, which were part of the compound discriminative stimuli.

Elemental visual discrimination task. The discriminative stimuli were identical with the elemental auditory discrimination task. Go responses to the discriminative stimuli that included light $\mathrm{X}(\mathrm{A}+\mathrm{X}, \mathrm{B}+\mathrm{X})$ and no-go responses to the discriminative stimuli that included light $\mathrm{Y}(\mathrm{A}+\mathrm{Y}, \mathrm{B}+\mathrm{Y})$ were correct. The rat was required to discriminate the elemental visual stimuli, which were part of the compound discriminative stimuli.

Configural auditory-visual discrimination task. The discriminative stimuli were identical with the elemental auditory and elemental visual discrimination tasks. Go responses to the discriminative stimulus that included both tone $\mathrm{A}$ and light $\mathrm{X}(\mathrm{A}+\mathrm{X})$ and no-go responses to the other discriminative stimuli were correct. The rat was required to discriminate the specific compound stimulus from the other discriminative stimuli.

Sequential auditory-visual discrimination task. The discriminative stimuli were sequential, not simultaneous, presentations of auditory and visual stimuli - that is, they were sequentially presented in that order, with a $1-\mathrm{sec}$ interstimulus interval $(A \rightarrow X$, $\mathrm{A} \rightarrow \mathrm{Y}, \mathrm{B} \rightarrow \mathrm{X}, \mathrm{B} \rightarrow \mathrm{Y}$ ). Go responses to the discriminative stimulus that included both tone $\mathrm{A}$ and light $\mathrm{X}(\mathrm{A} \rightarrow \mathrm{X})$ and no-go responses to the other discriminative stimuli were correct. The rat was required to discriminate the specific sequential stimulus from the other discriminative stimuli. This task alone needed temporal processing between the elemental auditory and visual stimuli.

Figure 1 illustrates the apparatus, the sequence of events in a trial, and the discriminative stimuli and correct responses in each of the tasks.

\section{Procedure}

Training. First, the rats were trained to the criterion ( $80 \%$ correct trials in a session) in the elemental auditory task, then trained to the criterion in the elemental visual task, then trained in the configural auditory-visual task, then trained in the sequential auditory-visual task, then trained in the elemental auditory task again, 


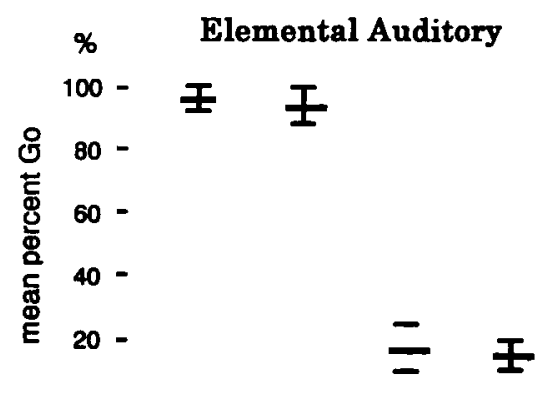

$A+X \quad A+Y \quad B+X \quad B+Y$

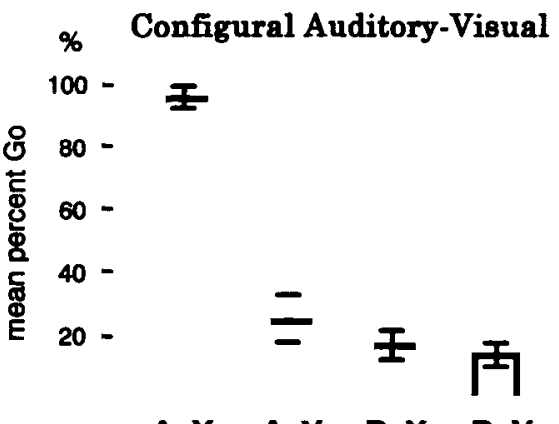

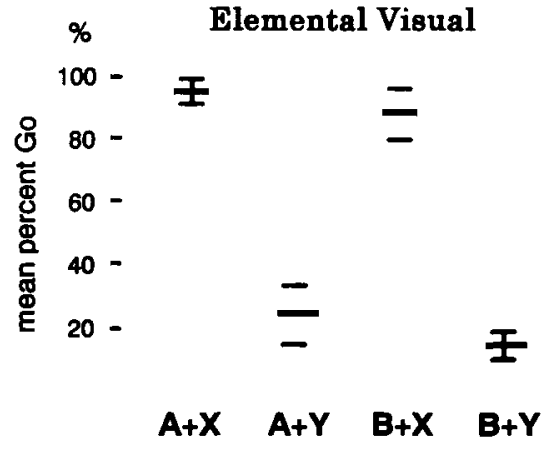

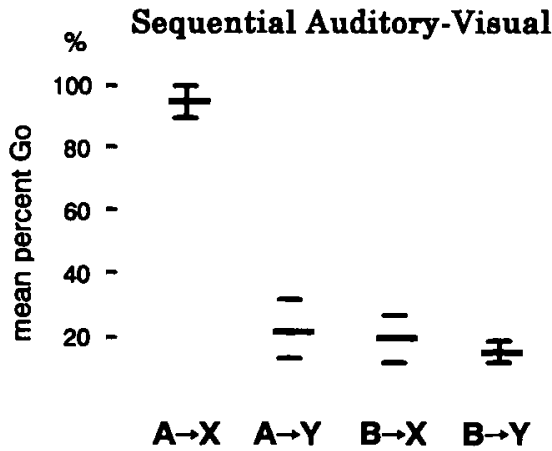

Figure 2. Mean go responses and their standard deviations for all the rats on the trials of each discriminative stimulus in each of the tasks when the training was completed, prior to the probe sessions.

and so forth. The training was continued until the rats reached the criterion in all tasks in 1 day. The order of the tasks in a day varied from day to day, and the interval between the tasks was around $2 \mathrm{~h}$.

Probe sessions of the elemental auditory discrimination. In the configural and sequential discrimination tasks, the rat had to discriminate specific compound $(\mathrm{A}+\mathrm{X})$ and sequential $(\mathrm{A} \rightarrow \mathrm{X})$ stimuli from other stimuli. In the elemental auditory discrimination task, however, the rat could perform correctly not only by discriminating a specific elemental stimulus (A) from another (B), but also by discriminating two specific compound stimuli $(A+X$ and $A+Y)$ from other compound stimuli $(B+X$ and $B+Y)$. In order to test the latter possibility, a session with probe trials was conducted after the training. In half of 80 trials with light $X$ and light $Y, X$ and $Y$ presentations were replaced by illuminations of a centrally located bulb (light $Z$ ) in a random order. Consequently, six discriminative stimuli $(\mathrm{A}+\mathrm{X}, \mathrm{A}+\mathrm{Y}, \mathrm{B}+\mathrm{X}, \mathrm{B}+\mathrm{Y}, \mathrm{A}+\mathrm{Z}, \mathrm{B}+\mathrm{Z})$ were presented in the trials, and the trials with $A+Z$ and $B+Z$ were probe trials.

Probe session of the elemental visual discrimination. A session with probe trials was conducted to test the possibility that the rat discriminated two compound stimuli $(\mathrm{A}+\mathrm{X}, \mathrm{B}+\mathrm{X})$ from other stimuli $(A+Y, B+Y)$ in the elemental visual discrimination task. In half of 80 trials with tone $A$ and tone $B, A$ and $B$ presentations were replaced by presentations of a middle tone $(6 \mathrm{kHz}$; tone $C)$ in a random order. Consequently, six discriminative stimuli $(\mathrm{A}+\mathrm{X}, \mathrm{A}+\mathrm{Y}$, $\mathrm{B}+\mathrm{X}, \mathrm{B}+\mathrm{Y}, \mathrm{C}+\mathrm{X}, \mathrm{C}+\mathrm{Y}$ ) were presented in the trials, and the trials with $\mathrm{C}+\mathrm{X}$ and $\mathrm{C}+\mathrm{Y}$ were probe trials.

\section{RESULTS}

All 10 rats reached the criterion performance in the elemental auditory discrimination task within 10 sessions of training. Next, 8 of the rats reached the criterion performance in the elemental visual discrimination task within 14 sessions. Two rats did not show stable performance even after 30 sessions of the elemental visual discrimination task and were removed from the experiment. Next, the remaining 8 rats reached the criterion performance in the configural auditory-visual discrimination task within 12 sessions. Finally, the 8 rats reached the criterion performance in the sequential auditory-visual discrimination task within 15 sessions. As training continued, with an alternation of all the tasks in 1 day, the rats gradually exhibited stable correct performance and finally showed performance greater than criterion in all the tasks within 15 days of the training. Figure 2 shows the mean go responses for the rats on all the types of trials in the sessions with all tasks when the training was completed, prior to the probe sessions.

Figure 3 shows the mean go responses for the rats on all the types of trials in the probe sessions of the elemental auditory discrimination task, in which the rats were required to conduct go responses to tone $A$, and in those of the elemental visual discrimination task, in which the rats were required to conduct go responses to light $\mathrm{X}$. There was no significant difference either among $A+X$, $\mathrm{A}+\mathrm{Y}$, and $\mathrm{A}+\mathrm{Z}$ stimuli or among $\mathrm{B}+\mathrm{X}, \mathrm{B}+\mathrm{Y}$, and $\mathrm{B}+\mathrm{Z}$ stimuli of the elemental auditory discrimination task; nor was there a significant difference either among the $\mathrm{A}+\mathrm{X}, \mathrm{B}+\mathrm{X}$, and $\mathrm{C}+\mathrm{X}$ stimuli or among the $\mathrm{A}+\mathrm{Y}, \mathrm{B}+\mathrm{Y}$, 

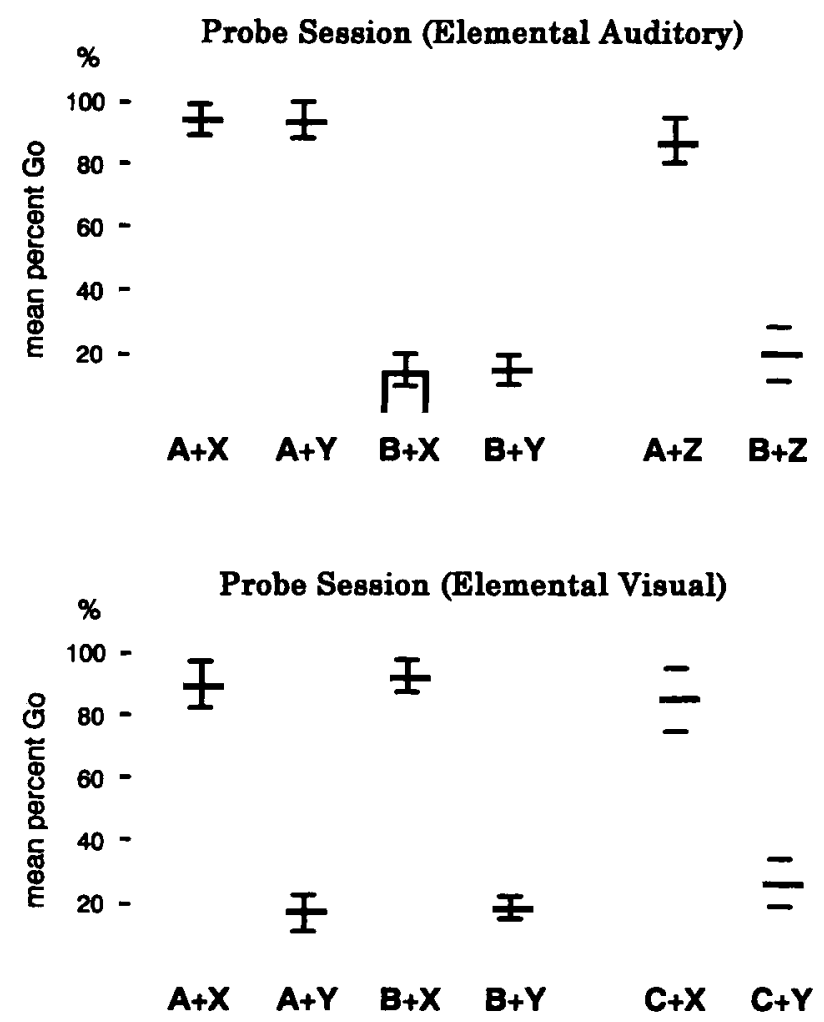

\begin{abstract}
Figure 3. Mean go responses and their standard deviations for all the rats on the trials of each discriminative stimulus in the probe session of the elemental auditory discrimination task and in that of the elemental visual discrimination task. The two bars at the far right in each panel are the results from the probe trials.
\end{abstract}

and $\mathrm{C}+\mathrm{Y}$ stimuli of the elemental visual discrimination task.

\section{DISCUSSION}

The present study shows that the elemental auditory, elemental visual, configural auditory-visual, and sequential auditory-visual discrimination tasks can be conducted in 1 day for a given rat after adequate training. Although it would be possible to simultaneously record many neuronal activities for several days or longer, given proper techniques, this result means that neuronal activity from a given neuron or a group of neurons can be recorded in all the tasks more easily and effectively within 1 day. This multiple-tasks comparison of neuronal activities will make it possible to see whether the view of cell assembly underlying memory processes is correct, by testing whether functional overlapping of the individual neurons and connection dynamics among the neurons are found when several different memories are being processed in the working brain.

A preliminary result (Sakurai, 1996a, 1998a) from a recording of hippocampal neuronal activities when rats were performing these kinds of elemental, configural, and sequential discrimination tasks showed that more neuron pairs showed activity correlation in the sequential discrimination task than in the other tasks. This suggests that the sequential discrimination task, which required temporal processing between elemental stimuli, might be unique and that the dynamics of neuronal correlation - that is, the dynamics of functional synaptic connections among neurons-might play especially important roles in the temporal processing of stimuli.

The stimuli were presented identically in the elemental auditory, elemental visual, and configural auditoryvisual tasks. Therefore, the cue for the rats that the tasks had changed was a difference in the response-reinforcer contingency. The rats had to make errors on the first several trials in a session to discover that the rules had changed. This means that the rats found the task demands by themselves. This is also the reason that the criterion for the learning was not very high $(80 \%)$ and that the first 20 trials were not included in the results in the probe sessions.

The results of the probe sessions of the elemental auditory discrimination showed that the probe trials - that is, replacing the familiar visual stimuli $(\mathrm{X}, \mathrm{Y})$ with an unfamiliar one (Z)--did not disrupt correct performance in the elemental auditory task. This means that the rats did not discriminate two compound stimuli $(\mathrm{A}+\mathrm{X}$ and $\mathrm{A}+\mathrm{Y})$ from the other two compound ones $(\mathrm{B}+\mathrm{X}$ and $\mathrm{B}+\mathrm{Y})$ but 
did, in fact, discriminate one elemental auditory stimulus (A) from the other elemental one (B). The probe sessions of the elemental visual discrimination also showed that the rats did discriminate one elemental visual stimulus (X) from the other elemental one (Y). It can be concluded that the elemental discrimination tasks are tasks in which elemental stimuli are discriminated.

This kind of behavioral analysis may become the basis for many kinds of pychobiological research, because the types of information being processed in the tasks employed are known - thus allowing for firm conclusions concerning the relationship between neural activities and cognitive functions. Also, establishing methods by which rodents may be trained with multiple and complex behavioral tasks is important for relating cognitive functions to the molecular findings of research in which rodents are the major experimental animal of choice.

\section{REFERENCES}

BARLOW, H. B. (1972). Single units and sensation: A doctrine for perceptual psychology? Perception, 1, 371-394.

Gothard, K. M., Skaggs, W. E., Moore, K. M., \& McNaughton, B. L. (1996). Binding of hippocampal CA1 neural activity to multiple reference frames in a landmark-based navigation task. Journal of Neuroscience, 16, 823-835.

HEBB, D. O. (1949). The organization of behavior: A neuropsychological theory. New York: Wiley.

PaLm, G. (1990). Cell assemblies as a guideline for brain research. Concepts in Neurosciences, 1, 133-147.

Pontecorvo, M. J. (1983). Effects of proactive interference on rats' continuous nonmatching-to-sample performance. Animal Learning \& Behavior, 11, 356-366.
SAKURAI, Y. (1987). Rats' auditory working memory tested by continuous non-matching-to-sample performance. Psychobiology, 15, 277 281.

SAKURAI, Y. (1990). Hippocampal cells have behavioral correlates during the performance of an auditory working memory task in the rat. Behavioral Neuroscience, 104, 253-263.

SakURAI, Y. (1992). Auditory working and reference memory can be tested in a single situation of stimuli for the rat. Behavioural Brain Research, 50, 193-195.

SAKURAI, Y. (1993). Dependence of functional synaptic connections of hippocampal and neocortical neurons on types of memory. Neuroscience Letters, 158, 181-184.

SAKURAI, Y. (1994). Involvement of auditory cortical and hippocampal neurons in auditory working memory and reference memory in the rat. Journal of Neuroscience, 14, 2606-2623.

SakURAI, Y. (1996a). Functional overlap and connectivity of hippocampal neurons in multiple discriminations of elemental, configural and successive stimuli in the rat. Society for Neuroscience $A b$ stract, 22, 914

SAKURA, Y. (1996b). Hippocampal and neocortical cell assemblies encode memory processes for different types of stimuli in the rat. Journal of Neuroscience, 16, 2809-2819.

SakURA, Y. (1998a). Cell-assembly coding in several memory processes. Neurobiology of Learning \& Memory, 70, 212-225.

SAKURAI, Y. (1998b). The search for cell assembly in the working brain. Behavioural Brain Research, 91, 1-13.

SAKURAI, Y. (1999). How do cell assemblies encode information in the brain? Neuroscience \& Biobehavioral Reviews, 23, 785-796.

Tanila, H., Shapiro, M. L., Gallagher, M., \& Eichenbaum, $H$. (1997). Brain aging: Changes in the nature of information coding by the hippocampus. Journal of Neuroscience, 17, 5155-5174.

(Manuscript received July 28, 1998 ; revision accepted for publication August 3, 1999.) 